\title{
Intenção de uso de comentários de viagem online na escolha de um meio de hospedagem: Fatores influenciadores
}

\author{
Intention to use online travel reviews in choosing an accommodation: \\ Influencing factors
}

\section{Intención de uso de comentarios de viaje online en la elección de un medio de hospedaje: Factores que influyen}

\author{
Marcela Martins Silva ${ }^{1}$ \\ Luiz Mendes Filho ${ }^{2}$
}

\begin{abstract}
Resumo: Cada vez mais os consumidores estão lendo e compartilhando comentários relacionados a viagem na Internet que são publicados por viajantes ao invés de consultarem informações geradas pelos próprios prestadores de serviços turísticos. O presente trabalho tem como objetivo analisar os fatores que influenciam a intenção de uso de Comentários de Viagens Online (CVO) na escolha de um meio de hospedagem. Para compreender quais são esses fatores influenciadores relacionados ao CVO no contexto brasileiro, o presente trabalho utiliza o Modelo de Aceitação Tecnológica e a Teoria Motivacional para dar o suporte na investigação. A metodologia sugerida foi a de cunho exploratório-descritivo, com abordagem quantitativa. A coleta de dados foi realizada com 53 estudantes universitários que já utilizaram o CVO na escolha de um meio de hospedagem. $\mathrm{O}$ estudo fez uso da técnica de Modelagem de Equações Estruturais Partial Least Squares (PLS), para testar o modelo proposto de oito hipóteses. Para avaliar as cinco variáveis do modelo de pesquisa (facilidade de uso, utilidade percebida, prazer percebido, atitude, e intenção), o PLS avaliou o modelo de mensuração e estrutural da pesquisa. Ao todo, cinco hipóteses foram confirmadas, e três foram rejeitadas. $\mathrm{O}$ que pode se concluir pelas hipóteses aceitas é que os viajantes entrevistados que acham útil o CVO, tem atitude e intenção de utilizá-lo na escolha de um meio de hospedagem.
\end{abstract}

Palavras-chave: Comentários de Viagem Online; Modelo de Aceitação Tecnológica; Teoria Motivacional; Meio de Hospedagem; Turismo.

Abstract: More and more consumers are reading and sharing travel-related comments on the internet that are published by travelers rather than consulting information generated by tourism service providers. The present study aims to analyze the influencing factors on the intention to use online travel reviews (OTR) in choosing an accommodation. To support the research, this study uses the Technology Acceptance Model and Motivational Theory to understand what the influencing factors are related to OTR in the Brazilian context. The suggested methodology was exploratory-descriptive with a quantitative approach. Data collection was performed with 53 university students who have used the OTR in choosing an

1 Mestranda do Programa de Pós-Graduação em Turismo da Universidade Federal do Rio Grande do Norte (UFRN).

Email:mar_celams@hotmail.com

2 Doutor em Administração pela Auckland University of Technology, Nova Zelândia. Professor do Departamento de Turismo e do Programa de Pós-Graduação em Turismo da UFRN. Email: luiz.mendesfilho@gmail.com 
accommodation. The Structural Equation Modeling technique Partial Least Squares (PLS) was used in this study to test the eight hypotheses proposed in the model. To evaluate the five variables of the research model (ease of use, perceived usefulness, perceived enjoyment, attitude and intention), the PLS evaluated the measurement and structural model of the research. As a result, five hypotheses were confirmed, and three were rejected. It can be concluded by the confirmed hypotheses is that travelers who consider OTR useful, they have attitude and intention to use it in choosing an accommodation.

Keywords: Online Travel Reviews; Technology Acceptance Model; Motivational Theory; Accommodation; Tourism.

Resumen: Cada vez más los consumidores están leyendo y compartiendo los comentarios relacionados con los viajes en Internet que son publicados por los viajeros en lugar de consultar la información generada por los prestadores de servicios turísticos. El presente trabajo tiene como objetivo analizar los factores que influyen en la intención de uso de Comentarios de Viajes Online (CVO) en la elección de un medio de hospedaje. Para comprender cuáles son esos factores que influyen relacionados con CVO en el contexto brasileño. Este estudio utiliza el Modelo de Aceptación de Tecnología y Teoría Motivacional par dar apoyo a la investigación. La metodología sugerida es de cuño exploratorio-descriptivo, con abordaje cuantitativo. La recolección de datos se llevó a cabo con 53 estudiantes universitarios que han utilizado el CVO en la elección de un medio de hospedaje. El estudio hizo uso de la técnica de Modelo de Ecuaciones Estructurales, Partial Least Squares (PLS), para poner a prueba el modelo propuesto de ocho hipótesis. Para evaluar las cinco variables del modelo de investigación (facilidad de uso, utilidad percibida, placer percibido, la actitud y la intención), el PLS evaluó el modelo de medición y estructural de la pesquisa. En total, se confirmaron cinco hipótesis, y tres fueron rechazadas. Lo que se puede concluir por las hipótesis aceptadas es que los viajeros encuestados a quienes les resulta útil el CVO, tiene la actitud y la intención de utilizarlo en la elección de un medio de hospedaje.

Palabras clave: Comentarios de Viaje Online; Modelo de Aceptación Tecnológica; Teoría de motivación; Medio de hospedaje; Turismo.

\section{INTRODUÇÃO}

As inovações tecnológicas estão transformando a maneira de distribuição dos serviços turísticos. Conforme Buhalis e Law (2008), se antes o viajante planejava sua viagem através de uma agência de turismo, com o advento da internet isso mudou. Os viajantes deixaram de atuar passivamente nesse processo para se tornar protagonistas dos mínimos detalhes de seu pacote de viagem obtidos através da Internet. A Internet possibilita maior interatividade, e as informações são visualizadas em forma de imagens, vídeos, textos, e comentários. Para Liu (2006), o "boca-a-boca" online desempenha um papel importante quando os consumidores precisam de informações para ajudá-los a decidir se a comprar um produto / serviço que eles não sabem bem.

$\mathrm{O}$ acesso à informação em tempo real online faz com que o viajante possa contratar os serviços necessários para seu turismo sem a necessidade dos intermediários. De acordo com o relatório do ITB World Travel Trend Report 2012/2013 (IPK International, 2012), os conteúdos gerados pelos usuários nas redes sociais estão ficando cada vez mais importante para ajudar e auxiliar os viajantes potenciais a planejarem suas viagens.

As pessoas podem planejar uma viagem acessando as diversas informações que a internet disponibiliza. Os viajantes estão usando as mídias sociais para falar sobre suas férias, mostrar fotos, trocar 
experiencias, buscar ideias sobre possiveis viagens, além de procurar opinioes e comentarios dos destinos, hoteis, atrações, e outros. A maneira que o conteúdo gerado pelos usuários influencia os viajantes a planejarem suas futuras viagens tem sido motivo de vários estudos. Por exemplo, na pesquisa realizada por Ayeh, Au e Law (2013b), viajantes potenciais consultam sites como TripAdvisor.com quando procuram Comentários de Viagens Online (CVO) para auxiliar em suas decisões de viagem e na formação de suas percepções e imagens de destinos. Os CVOs são comentários realizados por viajantes e disponibilizados na Internet, a respeito de produtos e serviços turísticos (Ayeh, Au \& Law, 2013a).

CVO é uma das formas mais importantes de mídia gerada pelo consumidor sobre experiência de viagens, uma vez que apresenta as opiniões dos viajantes sobre os lugares e os serviços por eles utilizados (Mendes-Filho, Tan, \& Mills, 2012; O'Connor, 2008; Vermeulen \& Seegers, 2009; Yoo \& Gretzel, 2011; Yoo \& Gretzel, 2008). Em um estudo conduzido por Gretzel, Yoo e Purifoy (2007) com viajantes online verificou-se que $77,9 \%$ acreditam que CVO foi extremamente ou muito importante para as decisões de acomodação, sendo esta a decisão mais importante relacionada a viagens dentre as opções pesquisadas. Muitos pesquisadores têm investigado o tema no exterior, como Ayeh et al. (2013a), Ayeh et al. (2013b), Jalilvand e Samiei (2012), e Burgess et.al (2009). Por exemplo, Filieri e Mcleay (2013), investigaram o que influencia os viajantes a adotarem informações dos CVO na tomada de decisão.

Para compreender como o CVO é utilizado no contexto brasileiro, o presente trabalho utiliza o Modelo de Aceitação Tecnológica (Davis, 1989) e a Teoria Motivacional (Davis, Bagozzi, \& Warshaw, 1992) para dar o suporte na investigação de quais fatores influenciam o viajante na intenção de uso do CVO na escolha de meio de hospedagem. Há algumas pesquisas na área da tecnologia e comportamento do consumidor utilizando modelos como o Modelo de Aceitação Tecnológica e a Teoria Motivacional. Entre esses pesquisadores encontra-se o Morosan e Jeong, (2008), e Casaló, Flavián, e Guinalíu (2010). No contexto de Hong Kong, Ayeh et al. (2013a), pesquisaram a intenção dos viajantes de utilizar o CVO no planejamento de viagens.

O presente estudo é classificado como descritivo-exploratório, e busca entender o seguinte problema de pesquisa: quais são os fatores que influenciam a intenção de uso de CVO na escolha de um meio de hospedagem? Desta forma, este trabalho tem como objetivo analisar os fatores que influenciam a intenção de uso de CVO na escolha de um meio de hospedagem, através da Teoria Motivacional e do Modelo de Aceitação Tecnológica.

\section{COMENTÁRIOS DE VIAGENS ONLINE (CVO)}

Os Comentários de Viagens Online (CVO) permitem aos viajantes compartilhar suas experiências de viagens com outras pessoas (Arsal, Backman \& Baldwin, 2008). Isso tem contribuído para a difusão do "boca-a-boca" online. Segundo Norman, Buhalis, e Law (2008) os viajantes consideram as comunicações online (Internet) como substituto ou um complemento da comunicação escrita.

Os consumidores satisfeitos impactam diretamente sobre a reputação da marca (exemplo: nome do hotel), ao espalhar suas experiências online, o que é equivalente à propaganda denominada "boca-aboca". O "boca-a boca" online pode ser entendido como qualquer afirmação positiva ou negativa feita por 
potencial, atual, ou ex-clientes sobre um produto, serviço ou empresa, disponível para uma grande variedade de pessoas através da Internet (Hennig-Thurau, Gwinner, Walsh, \& Gremler, 2004).

Jeong e Jeon (2008) constataram que o processo de decisão no planejamento de viagem é influenciado por CVO sobre suas experiências em relação aos serviços prestados, as instalações do meio de hospedagem e etc. O hotel, por exemplo, precisa reconhecer o papel da internet, através das mídias sociais e do conteúdo gerado pelo usuário, para desenvolver seu negócio e fidelizar os consumidores ( $O^{\prime}$ Connor, 2008).

Os comentários de viagem na internet habilitam os viajantes a tomar suas próprias decisões sobre o consumo dos serviços e produtos a serem adquiridos (Mendes-Filho \& Carvalho, 2014). Vários são os motivos pelos quais as pessoas geram os comentários de viagem na internet, George e Scerri (2007) citam que é para o próprio entretenimento, para analisar, avaliar e comentar sobre os serviços e produtos, etc. De acordo com George e Scerri (2007) essa nova realidade tem gerado como resultado um poder sem precedentes as pessoas, em relação a disponibilidade de conhecimento, informação sobre diferentes produtos e serviços.

Nesse contexto o CVO é um feedback real e atual, no qual os hotéis podem saber como anda seu empreendimento, e assim poder otimizar os pontos positivos e minimizar e corrigir os pontos negativos. De acordo com Ye, Law e Gu (2009) os CVOs positivos podem aumentar significativamente o número de reservas em um hotel. Portanto, a internet prepara os hotéis a partir dos CVO para enfrentar o mercado, melhorar a qualidade dos serviços/produtos, além de ajudar na busca do seu objetivo principal que é a obtenção de lucros e redução de custos.

\section{MODELO DE ACEITAÇÃO TECNOLÓGICA (TAM)}

O Modelo de Aceitação Tecnológica, do inglês Technology Acceptance Model (TAM), proposto por Davis (1989), tem sido utilizado para explicar a aceitação de novas tecnologias da informação e suas aplicações - ver Figura 1. De acordo com Stair e Reynolds (2006), o TAM investiga o conjunto de mecanismos envolvidos no uso de determinada tecnologia, que abarca desde sua facilidade de uso, sua utilidade até o grau de apoio da organização para o uso de um sistema de informações. O modelo TAM, em síntese, propõe que a intenção de adotar um dado sistema depende da atitude para sua utilização, que reflete as avaliações comportamentais favoráveis ou desfavoráveis do consumidor, e de sua utilidade e facilidade percebida do uso do sistema (Davis, 1989).

Figura 1 - Modelo de Aceitação Tecnológica

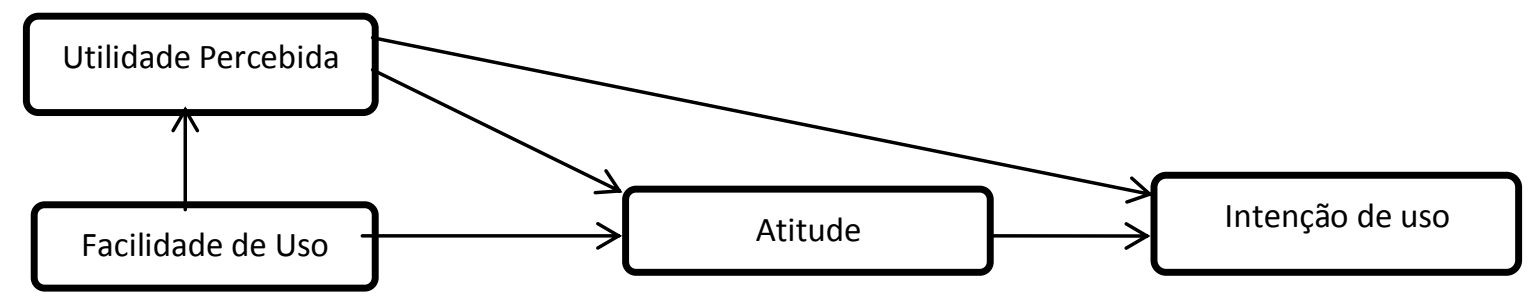

Fonte: Adaptado de Davis (1989) 
Este modelo busca explicar o que determina um indivíduo a rejeitar ou aceitar determinada tecnologia, sendo dada de forma imposta ou voluntária. As setas representam as relações causais dos constructos. A finalidade do autor Davis (1989) foi criar um modelo que verifique o comportamento real da utilização da tecnologia, através de estudos e análise das atitudes dos indivíduos, através da percepção de utilidade e da facilidade de usar.

Utilidade percebida é definida por Davis (1989) como o grau de que uma pessoa acredita que o uso de um sistema específico aperfeiçoará o seu trabalho. Nesse estudo, a utilidade descreve a expectativa do viajante de que usar o CVO pode otimizar seu plano de viagem. Vários estudos confirmaram a importância desse constructo na utilização da tecnologia em empreendimentos turísticos, e hotelaria (Huh, Kim, \& Law, 2009).

A utilidade é uma hipótese que pode influenciar a atitude e intenção dos viajantes em utilizar o CVO. O relacionamento entre atitude-utilidade é apoiado por uma série de estudos em outros contextos (Huh et al., 2009). Diante disso, propõe a hipótese:

H1 - Utilidade percebida de uso do CVO influencia positivamente a atitude em utilizá-lo na escolha de um meio de hospedagem.

O modelo prevê que há uma ligação direta entre a utilidade e a intenção. Essa relação está no princípio de que as pessoas desenvolvem suas intenções nas atitudes que considera útil. A relação foi confirmada por outros estudos (Casaló et al., 2010). Casaló et al. (2010) em seu estudo argumenta que a atitude está diretamente relacionada entre a utilidade e a intenção dos individuais em participar em comunidades de viagem online terceirizadas. Em vista disso, a seguinte hipótese é proposta:

H2 - Utilidade percebida de uso do CVO influencia positivamente a intenção de utilizá-lo na escolha de um meio de hospedagem.

A percepção facilidade de uso é outro fator fundamental em predição (predizer) no modelo de Davis (1989). O autor sugere que os indivíduos podem adotar a tecnologia se esta for percebida fácil de usar, ou seja, requerendo o mínimo esforço para sua realização. A influência da facilidade de uso no contexto do TAM foi validada em pesquisas anteriores (Castañeda, Frías, Munoz-Leiva, \& Rodrigues, 2007).

No contexto desse estudo, a facilidade é utilizada será entendida para explicar se o indivíduo acredita que ao usar o CVO está isenta de esforços. Por exemplo, ao escolher um meio de hospedagem, o hóspede potencial terá várias informações a mão, porém ele considerará a mais fácil de usar. Acredita-se que o CVO otimiza a conveniência em busca de informações e planejamento de viagem.

Portanto, a percepção de facilidade do viajante em utilizar o CVO pode influenciar sua atitude e sua percepção de utilidade na escolha do hotel (Davis, 1989). Diante dessa contextualização segue as hipóteses:

H3 - Facilidade percebida de usar o CVO influencia positivamente utilidade percebida na escolha de um meio de hospedagem.

H4 - Facilidade percebida de usar o CVO influencia positivamente atitude em utilizá-lo na escolha de um meio de hospedagem. 
Atitude foi definido Ajzen (1989, p. 241) como uma disposição do indivíduo "a responder favoravelmente ou desfavoravelmente a um objeto, pessoa, instituição ou evento". No caso deste estudo, o constructo atitude faz alusão à atitude do viajante em utilizar o CVO ao escolher um meio de hospedagem. O relacionamento entre atitude em relação ao um dado objeto ou comportamento e intenção comportamental é evidente em modelos utilizados no turismo e hospitalidade (Morosan et al., 2008). Portanto, este estudo tem a seguinte hipótese:

H5 - Atitude em usar o CVO influencia positivamente a intenção de utilizá-lo na escolha de um meio de hospedagem.

\subsection{Teoria Motivacional}

Da primeira ideia de se realizar uma viagem até finalmente o dia da viagem em si, pode-se levar muito tempo, sobretudo se essa viagem for motivada pelo lazer. Nesse meio tempo, a consulta a sites de viagens, redes sociais e outros meios que se associam a essa prática, vinculam-se essencialmente a fatores como a motivação e o prazer em si de realizar aquela ação, de apreciar os sites que interajam com o sujeito trazendo a ele o cenário de sua possível realização (Ayeh et al., 2013a).

A Teoria Motivacional, proposta por Davis et al. (1992), define que o comportamento dos usuários de Internet pode variar dependendo de sua motivação extrínseca ou intrínseca. A motivação extrínseca está associada à tomada de atitude visando à obtenção de recompensas específicas (Deci \& Ryan, 1987), sendo determinada por fatores externos. Por outro lado, a motivação intrínseca se associa à satisfação, ao prazer que o usuário tem ao realizar aquela atitude e está ligado a fatores internos (Vallerand, 1997).

Quando se trata da procura de informações de viagens, Castañeda et al. (2007), afirmam que o viajante pode está mais interessado na motivação intrínseca (ou seja, o prazer percebido do viajante ao obter uma informação), do que na motivação extrínseca. Para este estudo, a variável "prazer percebido" é utilizada para capturar essa motivação intrínseca.

Ryan e Deci (2001) afirmam que a níveis mais alto de motivação intrínseca os indivíduos tendem a gastar mais tempo em uma dada tarefa, e como resultado formar a percepção de facilidade de uso. E nesse caso, os indivíduos mais motivados estão mais propensos a minimizar a dificuldade em usar uma tecnologia. Em outros estudos constatou-se que o prazer percebido influencia a atitude e intenção à compra online (Ha \& Stoel, 2009).

Desta forma, as seguintes hipóteses são propostas:

H6 - Prazer percebido influencia positivamente a facilidade percebida de usar o CVO na escolha de um meio de hospedagem.

H7 - Prazer percebido influencia positivamente a atitude de usar o CVO na escolha de um meio de hospedagem.

H8 - Prazer percebido influencia positivamente a intenção de usar o CVO na escolha de um meio de hospedagem.

Segue abaixo o modelo de pesquisa e suas respectivas hipóteses propostas no estudo. 
Figura 2 - Modelo de Pesquisa

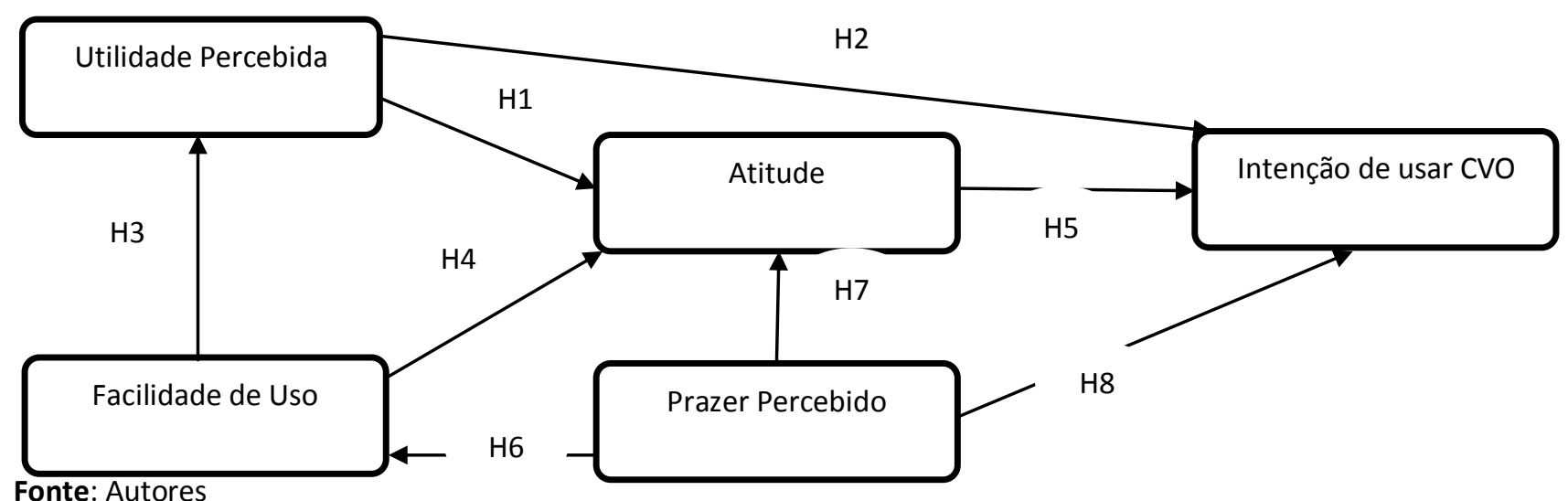

\section{METODOLOGIA}

O presente estudo tem o caráter exploratório-descritivo. Segundo Gil (2002), o estudo de caráter descritivo e exploratório tem o objetivo principal de aprimorar ideias e buscar informações sobre um determinado fenômeno. Por um lado, a pesquisa exploratória realiza descrições precisas e quer descobrir as relações existentes entre os componentes da mesma (Cervo \& Brevian, 2002). De acordo com Gil (2002), os dados da pesquisa são observados, registrados, analisados e correlacionados com os fatos ou fenômenos (variáveis) sem, entretanto, manipulá-los. Ainda segundo o autor, a pesquisa descritiva busca estudar fatos e fenômenos do mundo físico e humano sem a interferência do pesquisador.

Importante ressaltar que é utilizada também a pesquisa bibliográfica em livros, artigos científicos, e análise de trabalhos correlatos. Segundo Cervo e Brevian (2002), a pesquisa bibliográfica busca explicar um problema com base nas de referências teóricas publicadas em livros, artigos, teses, dentre outras. Ainda de acordo com os autores, a pesquisa bibliográfica é um procedimento necessário e básico para estudos acadêmicos, que tem como objetivo o domínio do "estado da arte" sobre certo tema.

A pesquisa tem abordagem quantitativa, na qual as informações geradas são traduzidas em números através da análise e interpretação de dados. São levantados os dados através de questionários e, por fim, transformados em dados estatísticos para entendimento do fenômeno de estudo. De acordo com Dencker (2000), faz-se necessária a utilização de técnicas e procedimentos estatísticos visando à facilidade de interpretação dos dados quando se trata de pesquisas de cunho quantitativo. A abordagem quantitativa adotada é semelhante às utilizadas em outros estudos, como por exemplo, Ryu, Kim e Lee (2009), e Venkatesh, Morris, Davis e Davis (2003) para predizer a intenção comportamental.

Para obtenção dos dados dessa pesquisa foram utilizados questionários. Os questionários foram aplicados durante o mês de fevereiro de 2014, com 53 estudantes universitários de graduação e pós-graduação que já utilizaram o CVO na escolha de um meio de hospedagem. Jovens universitários formam o típico perfil de usuário frequente da Internet e que leem comentários online quando estão fazendo o planejamento de viagens.

A escala utilizada no questionário é do tipo "likert" com respostas que irão desde discordo 
totalmente (1) até concordo totalmente (7). O questionário foi desenvolvido com base em medidas estabelecidas de construções a partir da literatura de marketing e tecnologia (Davis, 1989; Davis et al., 1992), e nos modelos já elaborados por pesquisadores de turismo (Ayeh et al., 2013a).

A análise dos dados é realizada através da Modelagem de Equações Estruturais, utilizando a técnica chamada Partial Least Squares (PLS). O software utilizado para avaliar o modelo teórico foi o PLS-Graph (Chin, 2010). O motivo da escolha do PLS, ao invés de outras técnicas de Modelagem de Equações Estruturais como LISREL e AMOS, foi devido ao tamanho da amostra do estudo piloto ser constituída apenas de 53 respondentes.

De acordo com Chin (1998), pode-se utilizar o PLS para análise de amostras com tamanho aceitável de 10 casos por indicador daquela variável latente que tenha o maior número de indicadores. Nesta pesquisa, a maior variável latente possui três indicadores, o que multiplicando-se por 10 obtêm-se um tamanho de amostra de 30 respondentes. Para este estudo foi utilizado 53 respondentes, portanto, suficiente para análise com PLS. As significâncias estatísticas dos coeficientes do modelo foram testadas utilizando-se a técnica bootstrapping disponível no PLS-Graph, com 500 amostras geradas a partir da amostra original.

\section{RESULTADOS E DISCUSSÕES}

Pelo perfil dos 53 respondentes, verifica-se em sua maioria ser do gênero feminino (68\%), e entre 18 e 34 anos (75\%). Com relação ao grau de escolaridade, $47 \%$ possuem ensino superior incompleto, $13 \%$ superior completo, e $39 \%$ pós-graduação. No que diz respeito a experiência com a Internet, cerca de $73 \%$ tem mais de 8 anos de uso dessa tecnologia. Em resumo, verifica-se que a maioria dos entrevistados são mulheres, jovens e como uma considerável familiarização com a Internet e uso do CVO na escolha de um meio de hospedagem.

A Tabela 1 apresenta dados referente a quantidade de CVO que se utiliza para escolher o estabelecimento hoteleiro. Pouco mais de $70 \%$ dos respondentes utilizam de 1 a 10 comentários para escolher uma hospedagem, e cerca de 19\% utilizam de 11 a 30 comentários de viagens online.

Tabela 1 - Quantidade de CVOs utilizados na escolha de um meio de hospedagem

\begin{tabular}{lll}
\hline $\begin{array}{l}\text { Quantos Comentários de Viagem Online (CVO) você utiliza ao } \\
\text { escolher um meio de hospedagem? }\end{array}$ & Frequência & $\%$ \\
\hline De 1 a 5 & 18 & 33,96 \\
De 6 a 10 & 20 & 37,73 \\
De 11 a 15 & 6 & 11,32 \\
De 16 a 20 & 3 & 5,67 \\
De 21 a 25 & 1 & 1,89 \\
De 26 a 30 & 0 & 0 \\
Mais de 30 & 5 & 9,43 \\
\hline
\end{tabular}

Fonte: Autores

Com relação à média do tempo de uso de CVO ao fazer a escolha de um meio de hospedagem (Tabela 2 ), verifica-se através da tabela 2 , que aproximadamente $51 \%$ dos respondentes utilizam menos de 
meia hora para realizar essa tarefa, 30\% de $1 / 2$ hora a 1 hora, $13 \%$ de 1 a 2 horas, e outros 5 gastam mais de 4 horas para escolher um meio de hospedagem.

Tabela 2 - Média do tempo do uso de CVO na escolha de um meio de hospedagem

\begin{tabular}{|c|c|c|}
\hline $\begin{array}{l}\text { Em média, quanto tempo você faz uso de Comentários de } \\
\text { Viagens Online (CVO) na escolha de um meio de } \\
\text { hospedagem? }\end{array}$ & Frequência & $\%$ \\
\hline Menos de meia hora & 27 & 50,94 \\
\hline De $1 / 2$ hora a 1 hora & 16 & 30,19 \\
\hline De 1 a 2 horas & 7 & 13,20 \\
\hline De 2 a 3 horas & 0 & 0 \\
\hline De 3 a 4 horas & 0 & 0 \\
\hline De 4 a 5 horas & 2 & 3,78 \\
\hline Mais de 5 horas & 1 & 1,89 \\
\hline
\end{tabular}

Fonte: Autores

Quanto aos websites utilizados para visualizar os CVOs na escolha de um meio de hospedagem (Tabela 3), o mais acessado foi o Decolar com 58\%, depois veio o TripAdvisor com $52 \%$, em seguida empatados o Booking e Facebook com 45\%. Também foram citados Hotéis e Malapronta como websites de CVO pelos respondentes.

Tabela 3 - Websites utilizados para visualizar o CVO na escolha de um meio de hospedagem

\begin{tabular}{lll}
\hline $\begin{array}{l}\text { Quais websites você utilizou os Comentários de Viagem Online } \\
\text { (CVO) ao escolher um estabelecimento hoteleiro? (Marque }\end{array}$ & & \\
tantos quantos se apliquem) & & \\
\hline Decolar & 31 & 58,49 \\
TripAdvisor & 28 & 52,83 \\
Booking & 24 & 45,28 \\
Facebook & 24 & 45,28 \\
Hotéis & 8 & 15,09 \\
Malapronta & 1 & 1,89 \\
\hline
\end{tabular}

Fonte: Autores

Em relação a quantidade de vezes que o entrevistado escreveu/postou um CVO sobre o meio de hospedagem após sua viagem (Tabela 4), consta-se que aproximadamente $37 \%$ dos respondentes nunca postaram nenhum CVO, 34\% raramente postam, outros $11 \%$ ocasionalmente comentam, e apenas $9 \%$ às vezes comentam e $7 \%$ frequentemente ou todas as vezes que viaja postam CVO.

Tabela 4 - Quantidade de vezes que escreveu/postou CVO após a sua viagem

\begin{tabular}{lll}
$\begin{array}{l}\text { Quantas vezes você costuma postar/escrever Comentários } \\
\text { de Viagem Online (CVO) após utilizar um meio de }\end{array}$ & $\%$ \\
hospedagem? & 20 & \\
\hline Nunca & 18 & 37,73 \\
Raramente & 6 & 33,96 \\
Ocasionalmente & 5 & 11,32 \\
Às vezes & 2 & 9,43 \\
Frequentemente & 0 & 3,78 \\
Muito frequentemente & 2 & 0 \\
Todas as vezes que eu viajo & 2 & 3,78 \\
\hline
\end{tabular}

Fonte: Autores 
Com o objetivo de testar o modelo de pesquisa foi utilizado o Partial Least Squares (PLS), através do software PLS-Graph, versão 3.0 (Chin, 2010). O PLS foi utilizado para avaliar a mensuração das variáveis (Tabelas 5 e 6), e o modelo estrutural e suas hipóteses (Figura 3).

Para avaliar as cinco variáveis do modelo de pesquisa (facilidade de uso, utilidade percebida, prazer percebido, atitude, e intenção) são verificados os carregamentos fatoriais e estatística " $t$ " (Tabela 5) de cada indicador de cada uma das variáveis, como também a validade convergente e confiabilidade composta das variáveis (Tabela 6).

A Tabela 5 apresenta os carregamentos fatoriais e estatística " $\mathrm{t}$ " das variáveis e seus indicadores. Os indicadores possuem um carregamento fatorial acima de 0,7, variando de 0,77 (mínimo) até 0,94 (máximo). De acordo com Chin (2010), os carregamentos fatoriais devem ser maiores que 0,7 para que os resultados sejam consideráveis bons e estatisticamente " $\mathrm{t}$ " significantes, acima de $5 \%$. Com relação a estatística " $\mathrm{t}$ ", verifica-se na tabela 5 , que as variáveis são significantes com o nível acima de $5 \%$. Os valores variaram de 5,00 (mínimo) até 57,43 (máximo).

Tabela 5 - Carregamentos fatoriais e Estatística t

\begin{tabular}{llll}
\hline Variáveis & Indicador & Carregamento & Estatística t \\
\hline \multirow{3}{*}{ Atitude } & AT1 & 0,8515 & 14,87 \\
& AT2 & 0,7795 & 5,00 \\
\multirow{4}{*}{ Intenção } & AT3 & 0,8365 & 10,24 \\
& IN1 & 0,8791 & 42,62 \\
& IN2 & 0,8911 & 18,07 \\
Utilidade & IN3 & 0,8512 & 12,31 \\
& UT1 & 0,8891 & 21,76 \\
Prazer Percebido & UT2 & 0,8351 & 9,45 \\
& UT3 & 0,8408 & 17,10 \\
& PP1 & 0,8647 & 22,58 \\
Facilidade & PP3 & 0,9312 & 57,43 \\
& FA1 & 0,7930 & 7,34 \\
& FA2 & 0,7793 & 10,61 \\
\hline
\end{tabular}

Fonte: Autores

Chin (2010) recomenda que a variância média extraída deve ser acima de 0,60, e a confiabilidade composta deve ser maior que 0,70 para se obter validade convergente. Pela Tabela 6 verifica-se que a variação média extraída foi acima de 0,68. Em relação a confiabilidade composta verifica-se também que as variáveis tiveram um resultado positivo, pois foi superior a 0,70 , sendo que a mínima atingida foi 0,86 e máxima foi 0,90 . Portanto, a variância média extraída e a confiabilidade composta foram validadas, pois se atingiu os limites aceitos (Chin, 2010). 
Tabela 6 - Variância Média Extraída e Confiabilidade Composta

\begin{tabular}{lll}
\hline Variáveis & Variância Média Extraída & Confiabilidade Composta \\
\hline Atitude & 0,68 & 0,86 \\
Intenção & 0,76 & 0,90 \\
Utilidade & 0,73 & 0,89 \\
Prazer Percebido & 0,75 & 0,90 \\
Facilidade & 0,70 & 0,87 \\
\hline
\end{tabular}

Fonte: Autores

O PLS foi utilizado para validar o modelo estrutural e suas hipóteses (Figura 3). Na avaliação utilizase os valores de $\mathrm{R}^{2}$ (variâncias) e das trilhas entre as hipóteses. De acordo com Chin (1998), valores de $\mathrm{R}^{2}$ próximos a $67 \%$ são substanciais, valores a cerca de $33 \%$ são considerados moderados, e os valores até $19 \%$ são considerados fracos. A Figura 03 também mostra as trilhas com seus valores de significância em relação a cada hipótese.

O modelo estrutural (Figura 03) explicou uma quantidade significativa na variância da intenção $\left(R^{2}=\right.$ $65 \%)$ e facilidade de uso $\left(R^{2}=53 \%\right)$ do CVO na escolha de um meio de hospedagem. As variâncias de utilidade percebida $\left(R^{2}=38 \%\right)$ e atitude $\left(R^{2}=30 \%\right)$ em utilizar o CVO foram considerados moderados no modelo estrutural. De um total de oito hipóteses propostas, cinco foram confirmadas $(\mathrm{H} 1, \mathrm{H} 2, \mathrm{H} 3, \mathrm{H} 5$, e $\mathrm{H6}$ ), e três foram rejeitadas ( $\mathrm{H} 4, \mathrm{H} 7$, e H8). As hipóteses confirmadas tiveram as trilhas estatisticamente significantes entre as variáveis, conforme discussão a seguir.

Figura 3 - Modelo Estrutural e Resultados

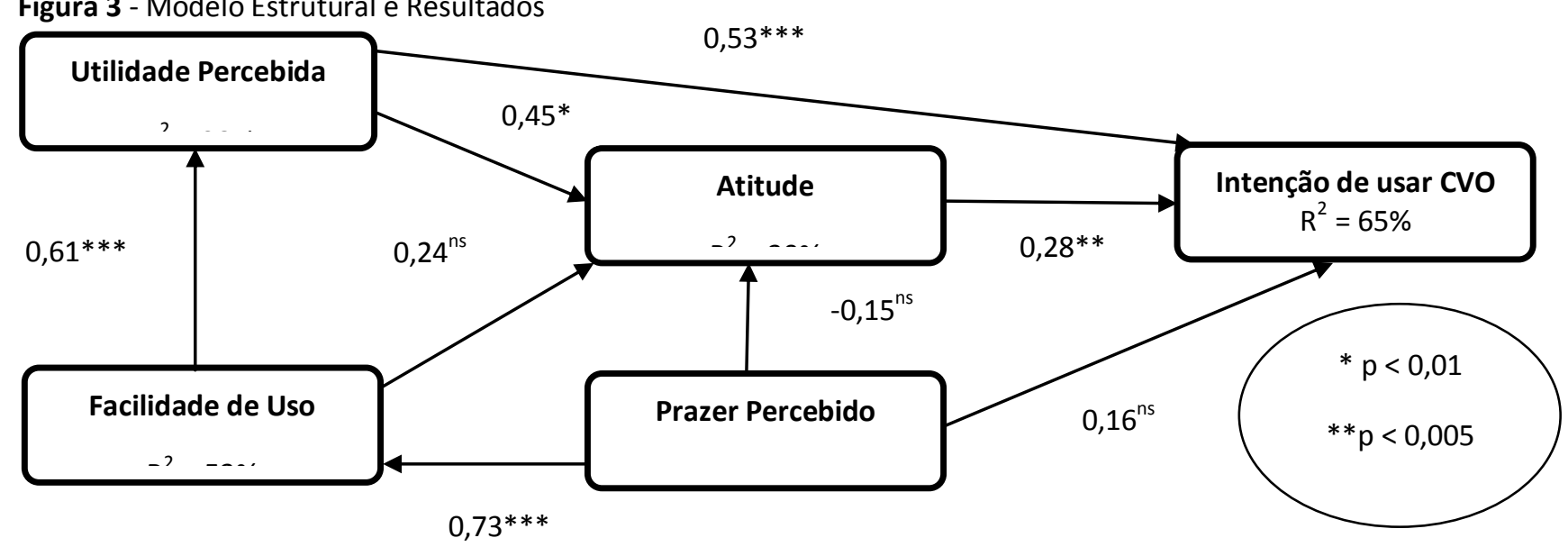

Fonte: Autores

A variável utilidade percebida de uso do CVO teve uma trilha estatisticamente significante para atitude de usar o CVO na escolha de um meio de hospedagem (trilha $=0,45, t=2,5, p<0,01$ ), confirmando a Hipótese 1. Portanto, essa afirmação confirma estudos já realizados por Ayeh et al. (2013a) e Huh et al. (2009), que atestam que a utilidade percebida tem impacto positivo em relação a atitude. A utilidade percebida de uso do CVO teve uma trilha estatisticamente significante para intenção (trilha $=0,53, t=3,8, p$ $<0,001$ ), o que confirma a Hipótese 2. Nessa perspectiva, verificou-se que é possível concordar com Kim, Lee, \& Law (2008), e Ayeh et al. (2013a), que constataram em suas pesquisas o efeito positivo da utilidade percebida sobre a intenção de uso. Desta forma, verifica-se que os viajantes que acham útil o CVO tem atitude e intenção de utilizar o CVO na escolha de um meio de hospedagem. 
Consistente com estudos de turismo anteriores (Ayeh et al., 2013a; Castañeda et al., 2007; Huh, et al., 2009), essa pesquisa confirma que a facilidade percebida de usar o CVO influencia a utilidade percebida (trilha $=0,61, t=6,3, p<0,001$ ) na escolha de um meio de hospedagem (Hipótese 3 ). Por outro lado, a Hipótese 4, onde foi testado se a facilidade percebida de usar o CVO influencia atitude, não foi confirmada (trilha $=0,24, t=0,7)$. O que contraria a pesquisa de Ayeh et al. (2013a), onde houve uma relação entre a facilidade de uso e a utilidade percebida de usar CVO para o planejamento de viagens em Hong Kong.

A Hipótese 5 foi confirmada, onde verificou-se que a atitude em usar o CVO influencia a intenção de utilizá-lo na escolha de um meio de hospedagem (trilha $=0,28, t=3,2, p<0,005$ ). Desta forma foi consistente com Ayeh et al. (2013a) e Ayeh et al. (2013b), onde também confirmaram que a atitude do viajante em usar o CVO tem influência na intenção de usá-lo na escolha de um meio de hospedagem.

Foi verificado que o prazer percebido tem impacto na facilidade percebida (Hipótese 6) de usar o CVO na escolha de um meio de hospedagem (trilha $=0,73, t=8,7, p<0,001$ ), o que é consistente com estudos anteriores sobre temática similares (Ayeh et al., 2013a; Ryu, et al., 2009). Porém, as hipóteses 7 e 8 foram rejeitadas, que verificaram respectivamente se o prazer percebido influencia a facilidade percebida de uso (trilha $=-0,15, t=0,5$ ), e intenção de usar o CVO (trilha $=0,16, t=1,7$ ) na escolha de um meio de hospedagem. $O$ que não corroborou com pesquisas de turismo anteriores (Ayeh et al., 2013a; Castañeda et al., 2007).

Tabela 7 abaixo mostra os resultados das hipóteses verificadas nesse estudo.

Tabela 7 - Resultado do teste das hipóteses

\begin{tabular}{lll}
\hline Hipótese & Resultados & Confirmação \\
\hline H1: Utilidade - Atitude & Trilha $=0,45, t=2,5, p<0,01$ & Sim \\
H2: Utilidade - Intenção & Trilha $=0,53, t=3,8, p<0,001$ & Sim \\
H3: Facilidade - Utilidade & Trilha $=0,61, t=6,3, p<0,001$ & Sim \\
H4: Facilidade - Atitude & Trilha $=0,24, t=0,7$ & Não \\
H5: Atitude - Intenção & Trilha $=0,28, t=3,2, p<0,005$ & Sim \\
H6: Prazer Percebido - Facilidade & Trilha $=0,73, t=8,7, p<0,001$ & Sim \\
H7: Prazer Percebido - Atitude & Trilha $=-0,15, t=0,5$ & Não \\
H8: Prazer Percebido - Intenção & Trilha $=0,16, t=1,7$ & Não \\
\hline
\end{tabular}

Fonte: Autores

\section{CONSIDERAÇÕES FINAIS}

Cada vez mais os consumidores estão lendo e compartilhando comentários relacionados a viagem na Internet que são publicados por pessoas próximas ao invés de consultarem as informações geradas pelos próprios prestadores de serviços turísticos (Gretzel et al., 2007). Em geral os viajantes usam o CVO durante a organização de sua viagem para consultar informações relativas ao destino a ser visitado, buscando informações sobre meios de hospedagem, restaurantes, etc (Mendes-Filho \& Carvalho, 2014; Gretzel \& Yoo, 2008).

O presente artigo contribui para o entendimento de que fatores influenciam a intenção de uso de CVO na escolha de um meio de hospedagem. Através de um modelo de pesquisa baseado em duas teorias (Modelo de Aceitação Tecnológica e Teoria Motivacional) e utilizando cinco variáveis (facilidade de uso, utilidade percebida, prazer percebido, atitude, e intenção), foram propostas oito hipóteses.

Ao todo, cinco hipóteses foram confirmadas $(\mathrm{H} 1, \mathrm{H} 2, \mathrm{H} 3, \mathrm{H} 5$, e H6), e três foram rejeitadas $(\mathrm{H} 4, \mathrm{H} 7$, e H8). Por este estudo, o que pode se concluir pelas hipóteses aceitas é que os viajantes entrevistados que 
acham útil o CVO, tem atitude e intenção de utilizá-lo na escolha de um meio de hospedagem. Além disso, o viajante que tem atitude em usar o CVO tem intenção de usá-lo para escolher o hotel que irá se hospedar. E aqueles viajantes que sentem "prazer" ao ler os CVOs tem mais facilidade de utilizá-lo no momento da escolha hoteleira. E essa facilidade de usar o CVO pelos viajantes entrevistados acaba influenciando a utilidade percebida de uso desses comentários online. Por outro lado, os viajantes que sentem "prazer" ao ler os CVOs não têm intenção e nem atitude de usá-los na escolha do hotel. Como também, aqueles viajantes que tiveram facilidade de utilizar o CVO, não necessariamente terão atitude de usá-lo ao escolher um estabelecimento hoteleiro.

Este estudo tem como contribuição acadêmica a utilização da Teoria Motivacional (Davis, Bagozzi, \& Warshaw, 1992) e Modelo da Aceitação Tecnológica (Davis, 1989) e a Teoria Motivacional (Davis et al., 1992) para ajudar na investigação de quais fatores influenciam o viajante na intenção de uso de CVO na escolha de um meio de hospedagem. Como contribuição prática da pesquisa, é possível afirmar que os CVOs estão tendo influência na decisão de escolha dos meios de hospedagem. Os empresários do setor de hospitalidade e turismo precisam estar atentos, pois um número crescente de viajantes está comprando pela Internet, sendo esta tendência influenciada pelo CVO (Litvin et al., 2008; Ye, Gu, \& Chen, 2011). Sendo assim, espera-se que os gerentes e executivos da indústria hoteleira verifique os CVOs que estão sendo escritos e postados nos websites e redes sociais pelos viajantes, para que se tome alguma posição sobre esses comentários negativos ou positivos a respeitos de seus estabelecimentos.

A pesquisa tem como principal limitação a população utilizada na coleta de dados e a amostra de apenas 53 respondentes. Por ser uma amostra pequena e de jovens universitários, não necessariamente os resultados obtidos com a Modelagem de Equações Estruturais refletem o perfil do viajante em geral que utiliza o CVO quando está escolhendo um meio de hospedagem. Esse estudo, portanto, é apenas preliminar. Pretende-se num futuro próximo realizar um survey com pelo menos 300 viajantes que utilizam CVO na escolha de meios de hospedagem. Outra sugestão de pesquisa futura a ser realizada, seria a utilização de métodos qualitativos para análise do uso do CVO na escolha de meio de hospedagem.

Por fim, conclui-se que intenção de uso de CVO na escolha de um meio de hospedagem vem influenciando cada vez mais os viajantes no processo de planejamento de viagens, e principalmente daqueles viajantes que utilizam websites e redes sociais no seu dia a dia.

\section{REFERÊNCIAS}

Arsal, L., Backamn, S., \& Baldwin, E. (2008). Influence of an online travel community on travel decisions. In P. O'Connor, W. Höpken, \& U. Gretzel (Eds.). Information and Communication Technologies in Tourism. (pp. 82-93). New York: Springer.

Ayeh, J. K., Au, N., \& Law, R. (2013a). Predicting the intention to use consumer-generated media for travel planning. Tourism Management, 35(1), 132-143.

Ayeh, J. K., Au, N., \& Law, R. (2013b). Do we believe in TripAdvisor? Examining credibility perceptions and online travelers' attitude toward using user-generated content. Journal of Travel Research, 52(4), 437-452. 
Buhalis, D., \& Law, R. (2008). Progress in information technology and tourism management: 20 years on and 10 years after the Internet: The state of e-tourism research. Tourism Management, 29(4), 609-623.

Casaló, L. V., Flavián, C., \& Guinalíu, M. A. (2010). Determinants of the intention to participate in firmhosted online travel communities and effects on consumer behavioral intentions. Tourism Management, 31(6), 898-911.

Castañeda, J. A., Frías, D. M., Munoz-Leiva, F., \& Rodriguez, M.A. (2007). Extrinsic and intrinsic motivation in the use of Internet as a tourist information source. International Journal of Internet Marketing \& Advertising, 4(1), 37-52.

Cervo, A.L., \& Bervian, P. A. (2002). Metodologia Científica (5a ed.). São Paulo: Prentice Hall.

Chin, W. W. (1998). Issues and opinion on structural equation modeling. MIS Quarterly, 22(1), vii-xvi.

Chin, W. W. (2010). How to write up and report PLS analyses. In V. E. Vinzi, W. W. Chin, J. Henseler, \& H. Wang (Eds.). Handbook of Partial Least Squares: Concepts, Methods and Applications. (pp. 655-690). New York: Springer.

Davis, F. D. (1989). Perceived usefulness, perceived ease of use, and user acceptance of information technology. MIS Quarterly, 13(3), 319-339.

Davis, F. D., Bagozzi, R. P., \& Warshaw, P. R. (1992). Extrinsic and intrinsic motivation to use computers in the workplace. Journal of Applied Social Psychology, 22(1), 1111-1132.

Deci, E. L., \& Ryan, R.M. (1987).The support of autonomy and the control of behavioral. Journal of Personality and Social Psychology, 53, 1024-1037.

Dencker, A. F. M. (2000). Métodos e técnicas de pesquisa em turismo (2a ed.). São Paulo: Futura.

Filieri, R., \& Mcleay, F. (2013). E-WOM and Accommodation: An Analysis of the Factors That Influence Traveler's Adoption of Information from Online Reviews. Journal of Travel Research, 53(1), 44-57.

George, C., \& Scerri, J. (2007). Web 2.0 and User-Generated Content: legal challenges in the new frontier. Journal of Information, Law and Technology, 12(2), 1-22.

Gil, A. C. (2002). Como elaborar projetos de pesquisa (4a ed.). São Paulo: Altas.

Gretzel, U., Yoo, K. H., \& Purifoy, M. (2007). Online travel review study: Role and impact of online travel reviews. Laboratory for Inteligent Systems in Tourism, Texas A\&M University. Recuperado em 20 de junho, 2014, de http://www.tripadvisor.com/pdfs/OnlineTravelReviewReport.pdf

Ha, S., \& Stoel, L. (2009). Consumer e-shopping acceptance: antecedents in a technology acceptance model. Journal of Business Research, 62, 565-571.

Hennig-Thuaru, T., Gwinner, K. P., Walsh, G., \& Gremler, D. D. (2004). Eletronic word-of-mouth via consumer opinion platforms: What motivates consumers to articulate themselves on the Internet? Journal of Interactive Marketing, 18(1), 38-52. 
Huh, H. J., Kim, T., \& Law, R. (2009). A comparasion of competing theoretical models for understanding acceptance behavior of information systems in upscale hotels. International Journal of Hospitality Management, 28(1), 717-724.

IPK International. (2012). ITB WORLD TRAVEL TRENDS REPORT 2012/2013. Berlim, Germany: Messe. Recuperado em 01 junho, 2014, de http://www.itbberlin.de/media/itbk/itbk media/itbk pdf/WTTR Report 2013 web.pdf

Jalilvand, M. R., \& Samiei, N. (2012).The impact of electronic word of mouth on a tourism destination choice: Testing the theory of planned behavior (TPB). Internet Research, 22, 591-612.

Jeong, M., \& Jeon, M.M. (2008). Customer reviews of hotel experiences through consumer generated media. Journal of Hospitality \& Leisure Marketing, 17, 121-138.

Kim, T. G., Lee, J. H., \& Law, R. (2008). An empirical examination of acceptance model. Tourism Management, 29, 500-513.

Lin, W.S. (2012). Perceived fit and satisfaction on web learning performance: IS continuance intention and task-technology fit perspectives. International Journal of Human -Computer Studies, 70, 498 -507.

Litvin, S. W., Goldsmith, R. E., \& Pan, B. (2008). Electronic word-of-mouth in hospitality and tourism management. Tourism Management, 29(3), 458-468.

Liu, Y. (2006). Word of mouth for movies: Its dynamics and impact on box office revenue. Journal of Marketing, 70(3), 74-89.

Mendes-Filho, L., \& Carvalho, M. S. D. (2014). Factores que influyen en el uso del contenido generado por el usuario en internet: Un estudio preliminar con viajeros brasileños. Estudios y Perspectivas en Turismo, 23(3), 607-625.

Mendes-Filho, L., Tan, F., \& Mills, A. (2012). User-generated content and travel planning: An application of the theory of planned behavior. Revista Brasileira de Pesquisa em Turismo, 6(3), 20-29

Moon, J. W., \& Kim, Y. G. (2001). Extending the TAM for word-wide-web context. Information \& Management, 38, 217-230.

Morosan, C., \& Jeong, M. (2008). Users'perceptions of two types of hotel reservation Web sites. International Journal of Hospitality Management, 27, 284-292.

Norman, A., Buhalis, D., \& Law, R. (2008). Complains on the online environment: The case of Hong Kong Hotels. In P. O'Connor, W. Höpken, \& U. Gretzel (Eds.). Information and Communication Technologies in Tourism. (pp. 73-85). New York, Springer.

O'Connor, P. (2008). User-Generated content and travel: A case study on TripAdvisor. In P. O'Connor, W. Höpken, \& U. Gretzel (Eds.), Information and Communication Technologies in Tourism. (pp. 47-58). New York, Springer.

Ryan, R. M., \& Deci, E. L. (2001). On happiness and human potentials: a review of research on hedonic and eudaimonic well-being. Annual Review of Psychology, 52, 141-166. 
Ryu, M. H., Kim, S., \& Lee, E. (2009). Understanding the factors affecting online elderly user's participation in video UCC services. Computers in Human Behavior, 25, 619 -632.

Stair, R. M., \& Reynolds, G. W. (2006). Princípios de Sistemas de Informação: uma abordagem gerencial. São Paulo: Pioneira Thomson Learning.

Vallerand, R. J. (1997). Toward a hierarchical model of intrinsic and extrinsic motivation. Advances in Experimental Social Pyschology, 29, 271-360.

Venkatesh, V., Morris, M. G., Davis, G.B., \& Davis, F. D. (2003). User acceptance of information technology: Toward a unified view. MIS Quarterly, 27(3), 425-478.

Vermeulen, I. E., \& Seegers, D. (2009). Tried and tested: The impact of online hotel reviews on consumer consideration. Tourism Management, 30(1), 123-127.

Ye, Q., Law, R., \& Gu, B. (2009). The impact of online user reviews on hotel room sales. International Journal of Hospitality Management, 28(1), 180-182.

Ye, Q., Law, R., Gu, B., \& Chen, W. (2011). The influence of user-generated content on traveler behavior: An empirical investigation on the effects of e-word-of-mouth to hotel online bookings. Computers in Human Behavior, 27(2), 634-639.

Yoo, K. H., \& Gretzel, U. (2011). Influence of personality on travel-related consumer-generated media creation. Computers in Human Behavior, 27(2), 609-621.

Yoo, K. H., \& Gretzel, U. (2008). What motivates consumers to write online travel reviews? Information Technology and Tourism, 10(4), 283-295.

Artigo recebido em: 20/05/2014.

Artigo aprovado em: 21/11/2014. 